\title{
Extended exposure to follicular fluid is required for significant stimulation of the acrosome reaction in human spermatozoa*
}

\author{
Caroline E. Stock $\uparrow$, R. Bates $\uparrow$, K. S. Lindsay $\uparrow$, D. K. Edmonds $\dagger$ and \\ Lynn R. Fraser
}

Chelsea Hospital for Women, Dovehouse Street, Londo. SW3 6LT, UK; and $\ddagger$ Department of Anatomy \& Human Biology, King's College London, Strand, London WC2R 2LS, UK

\begin{abstract}
Summary. The effect of human follicular fluid (FF) on the incidence of spontaneous acrosome reactions (AR) in human spermatozoa was examined over a $24-25$-h period using electron microscopy. Suspensions of motile spermatozoa were prepared by a swim-up method in Earle's medium, known to support in-vitro fertilization. After adjusting the concentration to $10 \times 10^{6} \mathrm{cell} / \mathrm{s} / \mathrm{ml}$, suspensions were diluted $1: 1$ with medium (control) or FF, the latter giving a final concentration of $50 \% \mathrm{FF}$. In addition, at $5 \mathrm{~h}$ and $24 \mathrm{~h}$ an aliquant of the control suspension was removed, diluted 1:1 with FF and incubated for $1 \mathrm{~h}$; the three suspensions were examined at $6 \mathrm{~h}$ and $25 \mathrm{~h}$. Continuous exposure to $50 \%$ FF stimulated the AR, the effect being significant $(P<0.001)$ at $25 \mathrm{~h}$. However, the 1-h short exposure of spermatozoa to FF did not produce an increase in $\mathrm{AR}$, even after $24 \mathrm{~h}$ preincubation. In a separate series of experiments, the effect of continuous incubation for $24 \mathrm{~h}$ in increasing concentrations of FF was investigated. A significant linear dose-dependent effect on the AR was observed with all concentrations assessed $(P<0.01$ for $12.5 \% \mathrm{FF}$ and $P<0.001$ for $25,50,75$ and $100 \% \mathrm{FF}$, compared with FF-free control). Therefore, human FF can stimulate the AR, but only after a continuous exposure to $\mathrm{FF}$. A short exposure to $\mathrm{FF}$, even after $24 \mathrm{~h}$ preincubation, does not trigger an increased $A R$ response.
\end{abstract}

Keywords: follicular fluid; human; acrosome reaction; electron microscopy

\section{Introduction}

Sperm capacitation and the acrosome reaction (AR) are essential prerequisites for successful mammalian fertilization. Freshly ejaculated spermatozoa are unable to fertilize oocytes, but they acquire functional competence following their removal from seminal plasma and sufficient incubation in permissive conditions, the length required being species-dependent (Bedford, 1970, 1983). Capacitation is a complex phenomenon involving changes in intracellular and surface components which prepare spermatozoa for the AR (Fraser \& Ahuja, 1988). The latter involves the structural breakdown of the plasma and outer acrosomal membranes, resulting in the release of lytic enzymes. Although early work on mammalian spermatozoa (Austin, 1951; Chang, 1951) suggested that the female genital tract was essential, the development of successful in-vitro fertilization systems has demonstrated that fertilization can take place outside the female.

\footnotetext{
* Reprint requests to Dr Lynn R. Fraser.

†Present address: Reproductive Medicine Unit, Queen Charlotte's Hospital, Goldhawk Road, London W6 0XG, UK.
} 
However, the factors which inititate and promote the AR are unclear, as is the physiological site where the reaction occurs.

Since in-vitro techniques have eliminated the requirement for the female tract and its fluids, the oocyte and its surrounding investments have attracted considerable attention. Meizel (1985) has reviewed the possible roles of molecules associated with the cumulus oophorus and/or the zona pellucida in triggering the AR, concluding that the AR could occur at several sites in response to different molecules. These molecules may work independently or synergistically to maximize the chances of fertilization, and although the female tract is not essential it may have a role to play. At ovulation, follicular fluid (FF), a complex fluid composed of secretions derived from both the follicle and plasma (Edwards, 1974), is released with the oocyte and may therefore be present at the site of fertilization in vivo. Furthermore, FF has been shown to stimulate the AR in numerous species including man (see 'Discussion'). Investigations using human FF and human spermatozoa have been limited due to the difficulty in obtaining fresh ovulatory FF, but the development of invitro fertilization techniques, including ovarian stimulation with exogenous hormones followed by aspiration of preovulatory follicles, has overcome this problem.

Using transmission electron microscopy to assess ultrastructural features of the AR in human spermatozoa, we previously reported a relatively low incidence $(\sim 15 \%)$ of spontaneous AR, even after prolonged incubation in a culture medium that supports in-vitro fertilization (Stock \& Fraser, 1987). The present study was designed to investigate whether FF, obtained from patients undergoing in-vitro fertilization treatment at Chelsea Hospital for Women, could stimulate the AR in human spermatozoa. A preliminary report of this study has been presented in abstract form (Stock et al., 1988).

\section{Materials and Methods}

\section{Follicular fluid preparation}

Follicular fluid (FF) was obtained from women attending the Chelsea Hospital for Women in-vitro fertilization programme, using the following protocol. Patients were superovulated with $50 \mathrm{mg}$ clomiphene citrate (Serophene; Serono Ltd, Welwyn Garden City, Herts, UK) and Pergonal (Serono Ltd) equivalent to 150 i.u. FSH intramuscularly (i.m.) from Days 3 to 7 of their cycle. From Day 8 onwards, follicular growth was monitored daily by ultrasonography and measurement of serum oestradiol. Human chorionic gonadotrophin ( 5000 i.u.) was given i.m. when there were 3 or more mature follicles (>12 mm diameter) and the leading follicle had reached $16 \mathrm{~mm}$. Oocyte retrieval and FF collection were carried out $35 \mathrm{~h}$ later using laparoscopy. Briefly, the ovary was held so that the follicles were conveniently positioned for puncture and, having activated suction, an aspirating needle punctured the follicle lateral to the thinnest point to avoid rupture and ensure a tight seal. The total fluid content was then aspirated and sent to the laboratory for oocyte identification; no heparin was used in aspirating the follicles. This procedure was repeated for all the major follicles. All samples of FF were labelled, centrifuged to remove blood and granulosa cells and the supernatant was stored at $-20^{\circ} \mathrm{C}$. Only follicular fluid obtained from patients with at least some oocytes that were subsequently fertilized in vitro were reserved for this study. To provide sufficient FF for these experiments pooled samples from the same patient were used as necessary.

\section{Sperm samples}

Human spermatozoa were obtained from men attending the Infertility Clinic or from men of proven fertility. The samples were collected by masturbation, after 3 days of abstinence, into a sterile container and allowed to liquefy for at least $30 \mathrm{~min}$. Each sample was then analysed and only samples with $>50 \%$ motile spermatozoa and sperm counts of $>60 \times 10^{6} \mathrm{cells} / \mathrm{ml}$ which were free of cellular contamination were used. Modified Earle's medium (Gibco Ltd, Cowley, Oxford, UK) with added gentamicin, which was able to support fertilization of $60-90 \%$ of human oocytes (unpublished observations), was used throughout this study. This medium was supplemented with $5 \mathrm{mg}$ Fraction $\mathrm{V}$ human serum albumin (hSA; Sigma, Poole, Dorset, UK) per ml.

\section{Sperm preparation and culture}

Series I. A swim-up technique was used to obtain a suspension of motile spermatozoa (Stock \& Fraser, 1987). For this, $1 \mathrm{ml}$ medium was pipetted into each of several sterile plastic 14-ml tubes (Falcon Plastics, Becton Dickinson UK 
Ltd, Cowley, Oxford, UK) and $0.5 \mathrm{ml}$ semen was then gently injected underneath. The tubes were sealed and incubated upright for $1-1.5 \mathrm{~h}$ at $37^{\circ} \mathrm{C}$. The motile spermatozoa were aspirated from the interface, pooled and sperm motility was evaluated. This technique produced a sperm population with a motility of $\geq 95 \%$. The sperm concentration was determined using a haemocytometer with spermatozoa that had been fixed in formol saline ( $4 \%$ formaldehyde) and the count was adjusted to $10 \times 10^{6} \mathrm{cells} / \mathrm{ml}$. If, however, the sperm concentration was less than $10 \times 10^{6} / \mathrm{ml}$, which was the case in 1 out of the 7 samples, the spermatozoa were centrifuged at $600 \mathrm{~g}$ for $15 \mathrm{~min}$ and resuspended in Earle's medium to a concentration of $10 \times 10^{6}$ cells $/ \mathrm{ml}$.

Each sperm suspension in Earle's medium was divided between two sterile Falcon tubes. To one an equal volume of Earle's medium was added (control suspension) and to the other an equal volume of $\mathrm{FF}$ was added ( $50 \% \mathrm{FF}$ final concentration). The final sperm concentration was $5 \times 10^{6} \mathrm{cells} / \mathrm{ml}$. Tubes were gassed with $5 \% \mathrm{CO}_{2}, 5 \% \mathrm{O}_{2}, 90 \%$ $\mathrm{N}_{2}$, capped and incubated at $37^{\circ} \mathrm{C}$. After $5 \mathrm{~h}$, an aliquant was removed from the control suspension, assessed for motility, diluted 1:1 with FF and incubated for a further $1 \mathrm{~h}$. After a total of $6 \mathrm{~h}$ the motility of all 3 suspensions (control, control $+1 \mathrm{~h} 50 \% \mathrm{FF}$ and continuous $50 \% \mathrm{FF}$ ) was assessed and an aliquant of each was fixed for electron microscopy. At $24 \mathrm{~h}$, another aliquant was removed from the control suspension, diluted $1: 1$ with FF and incubated a further $1 \mathrm{~h}$. Again, at $25 \mathrm{~h}$, aliquants from all 3 suspensions were assessed for motility and fixed. Seven semen samples were analysed.

Series $I I$. Motile sperm suspensions $(n=8)$ were prepared according to the procedure used in the in-vitro fertilization clinic. Unlike Series I and the normal clinic routine, however, the spermatozoa were not separated directly after liquefaction but remained in seminal plasma for a number of hours before processing; pressures of clinic duties necessitated this delay. The semen was diluted 1:1 with Earle's medium $(5 \mathrm{mg} \mathrm{hSA} / \mathrm{ml})$ and centrifuged at $600 \mathrm{~g}$ for $4 \mathrm{~min}$. The pellet was resuspended in $1 \mathrm{ml}$ Earle's medium and centrifuged for another $4 \mathrm{~min}$. The supernatant was gently aspirated and $1 \mathrm{ml}$ Earle's medium was carefully layered on to the pellet. Duplicate Falcon tubes were capped and incubated upright at $37^{\circ} \mathrm{C}$; after $30 \mathrm{~min} 0.5-0.75 \mathrm{ml}$ separated sperm suspension was aspirated from each and pooled. The motility and count were assessed and the sperm suspensions were adjusted to $10 \times 10^{6}$ cells $/ \mathrm{ml}$ with Earle's medium. Each suspension was cultured as described for Series I, except that only the longer incubation time of $25 \mathrm{~h}$ was examined.

Series III. Suspensions $(n=7)$ of motile spermatozoa were prepared as described in Series I. To ensure sufficient numbers of cells, only samples with high inital counts $\left(100-300 \times 10^{6} \mathrm{cell} / \mathrm{s} / \mathrm{ml}\right)$ were used and a slightly longer time (up to $2 \mathrm{~h}$ ) for swim-up was allowed. The final pooled motile suspension was diluted to $10 \times 10^{6} \mathrm{cells} / \mathrm{ml} ; 1 \mathrm{ml}$ was pipetted into each of 6 sterile $5-\mathrm{ml}$ Falcon tubes and centrifuged gently at $600 \mathrm{~g}$ for $5 \mathrm{~min}$. The supernatant was quickly but carefully removed and the pellet was resuspended in the appropriate volume of Earle's medium and/or FF to give final concentrations of $0,12 \cdot 5,25,50,75$ and $100 \% \mathrm{FF}$. The tubes were gassed, capped and incubated at $37^{\circ} \mathrm{C}$ for $24 \mathrm{~h}$; after assessment of motility, all were fixed.

\section{Electron microscopy and assessment}

The sperm suspensions were fixed in $3 \%$ glutaraldehyde in $0 \cdot 1 \mathrm{M}$-cacodylate buffer, $\mathrm{pH} 7 \cdot 2$, for $45 \mathrm{~min}$ (room temperature). Samples were then centrifuged at $600 \mathrm{~g}$ for $5 \mathrm{~min}$ and the pellet fixed in $0.1 \%$ osmium tetroxide and then washed in $50 \%$ alcohol. The pellet was 'blocked' in molten agar $(2 \%)$ and dehydrated through a series of graded alcohols and embedded in Araldite resin. Ultra-thin sections were cut with a glass knife, stained with uranyl acetate and lead citrate and examined with a Philips $301 \mathrm{G}$ or Hitachi HU12A electron microscope. A minimum of 200 sperm heads, over a number of random fields, was assessed in each sample; the same individual (C.E.S.) carried out all evaluations. Individual cells were classified into one of the 6 stages of the acrosome reaction which we have previously described (Stock \& Fraser, 1987). Stage I spermatozoa are unreacted, acrosome-intact cells. Cells at Stages $2-4$ are at intermediate stages, in which initiation of the acrosome reaction is evident, i.e. swelling of acrosomal cap, formation of intra-acrosomal vesicles primarily from outer acrosomal membrane, loss of acrosomal matrix but retention of outer acrosomal and plasma membranes. Spermatozoa at Stages $5+6$ are acrosome-reacted: the outer membranes have disappeared, although some vesicles may still be associated with the now exposed inner acrosomal membrane. Typical examples of Stages 1,3 and 6 are shown in Fig. 1. Only morphologically normal sperm heads were counted.

\section{Statistical evaluation}

Data were analysed using Cochran's modification of the $2 \times 2$ contingency tables (Snedecor \& Cochran, 1967). This test compares responses within individual replicates and therefore takes account of any variations among samples.

\section{Results}

\section{Series I}

Motile spermatozoa were incubated for a total of 6 and $25 \mathrm{~h}$ : (1) continuously in Earle's medium (control); (2) in Earle's medium for 5 or $24 \mathrm{~h}+1 \mathrm{~h}$ in $50 \% \mathrm{FF}$ (control $+1 \mathrm{~h} \mathrm{FF}$ ); con- 

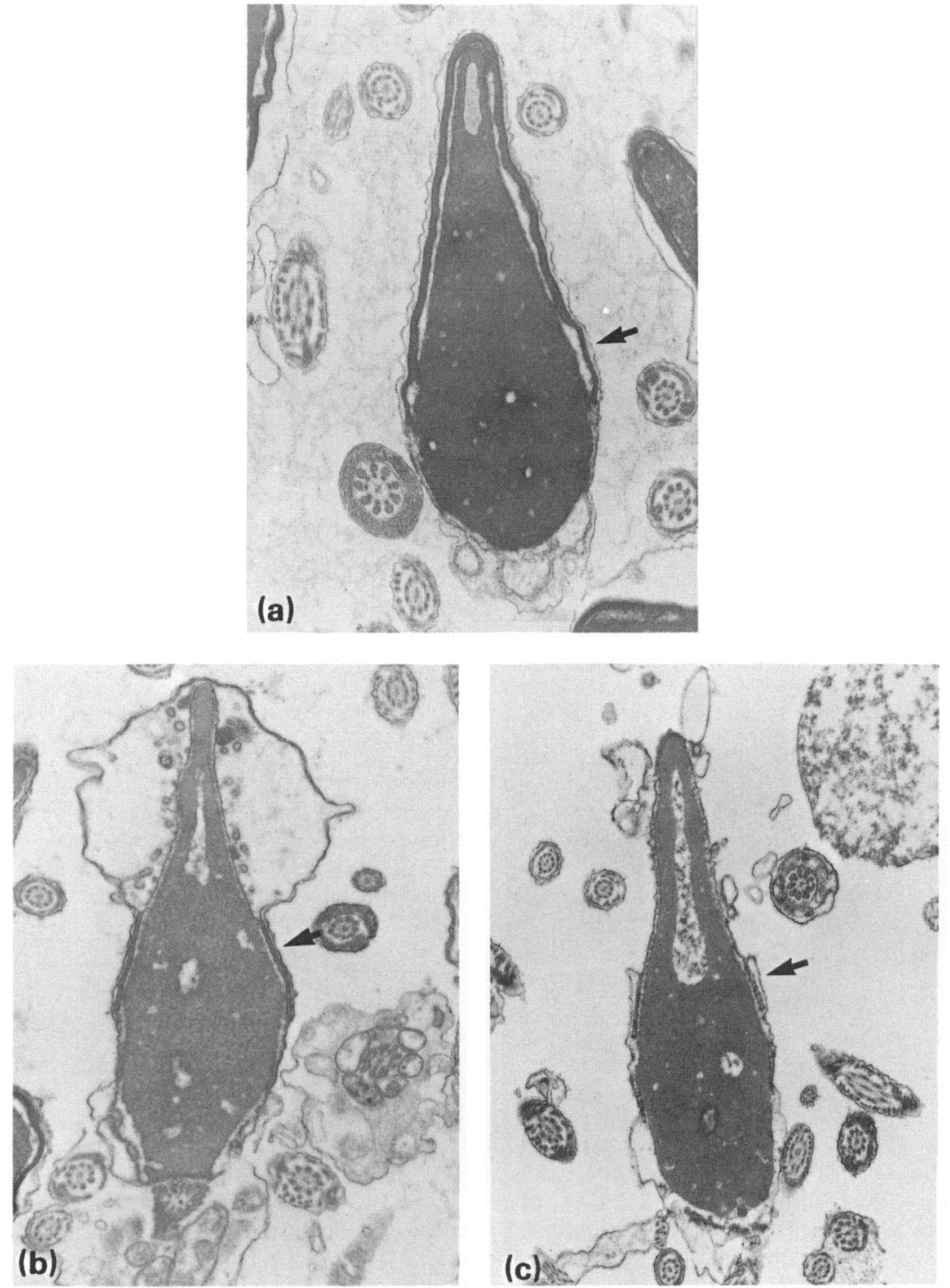

Fig. 1. Typical ultrastructural features of human spermatozoa, incubated in the presence or absence of human FF, at various stages of the AR. (a) Stage 1 (acrosome-intact); (b) Stage 3 (undergoing the AR, with diminished acrosomal matrix, intra-acrosomal vesicles present yet plasma and outer acrosomal membranes still distinguishable); (c) Stage 6 (acrosome-reacted, but still some associated membranous vesicles). The equatorial segment is indicated by an arrow. $\times 15000$.

tinuously in $50 \% \mathrm{FF}$ (continuous FF). After $6 \mathrm{~h}$ the total proportion of cells undergoing (Stages $2-$ 4) and having completed (Stages $5+6$ ) the AR was relatively low in all 3 groups: $9 \cdot 5 \%$, control; $8 \cdot 4 \%$, control $+1 \mathrm{~h} \mathrm{FF} ; 13 \cdot 3 \%$, continuous FF (Fig. 2a). Although the number of responding cells in the continuous FF group was higher (due to an increase in cells at intermediate stages) than in the other 2 groups, the differences were not statistically significant. After $25 \mathrm{~h}$ incubation, the proportion of reacted cells in all groups had increased: $15.0 \%$, control; $16.0 \%$, control $+1 \mathrm{~h} \mathrm{FF}$; $36.2 \%$, continuous FF (Fig. 2b). Values in the last group were significantly higher $(P<0.001)$ than 

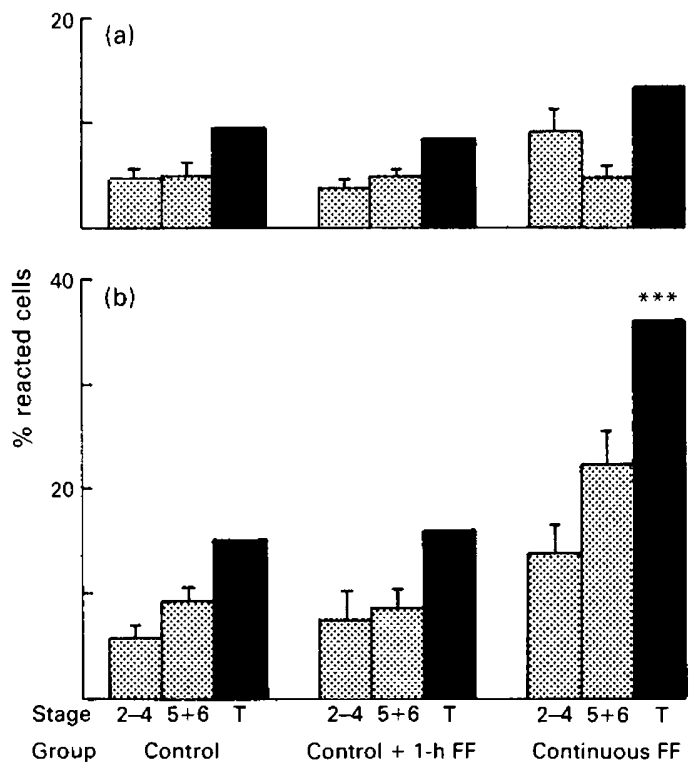

Fig. 2. AR response in human sperm suspensions $(n=7)$ prepared by swim-up and incubated a total of (a) $6 \mathrm{~h}$ and (b) $25 \mathrm{~h}$ : continuously in Earle's medium (control); in Earle's medium $+1 \mathrm{~h}$ in $50 \%$ human FF (control $+1 \mathrm{~h} \mathrm{FF}$ ); continuously in $50 \% \mathrm{FF}$ (continuous FF). The stippled bars represent the proportion of cells at Stages $2-4$ and $5+6$ (mean $\% \pm$ s.e.m.). The solid bars (T) represent the total mean reacted cells (Stages $2-6) .{ }^{* * *} P<0.001$ compared with other suspensions.

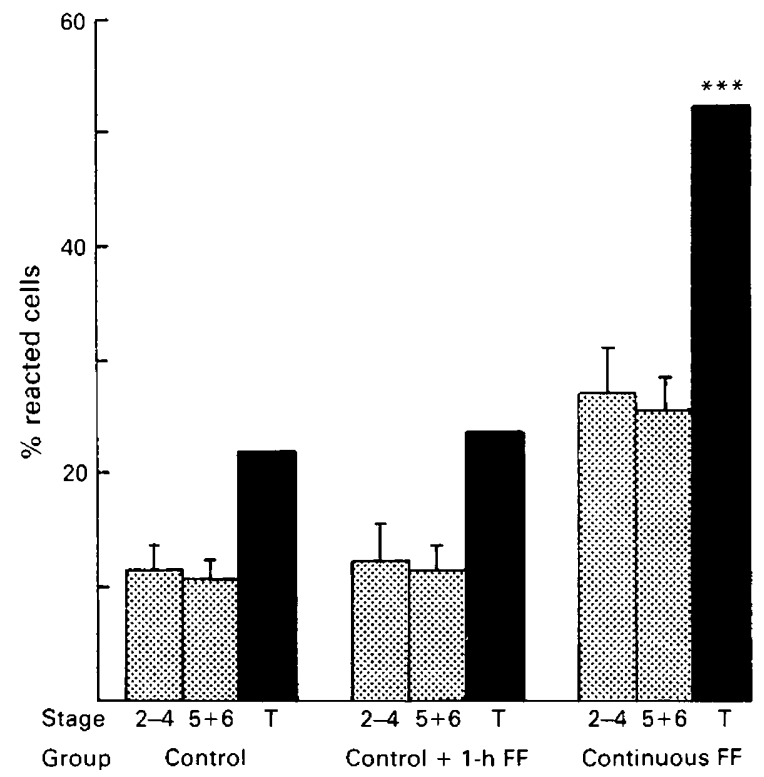

Fig. 3. AR response in human sperm suspensions $(n=8)$ prepared by centrifugation and swimup and incubated for $25 \mathrm{~h}$ : continuously in Earle's medium (control); in Earle's medium for $24 \mathrm{~h}+1 \mathrm{~h}$ in $50 \%$ FF (control $+1 \mathrm{~h}$ FF); continuously in $50 \%$ FF (continuous FF). ***P $<0.001$ compared with other suspensions. 

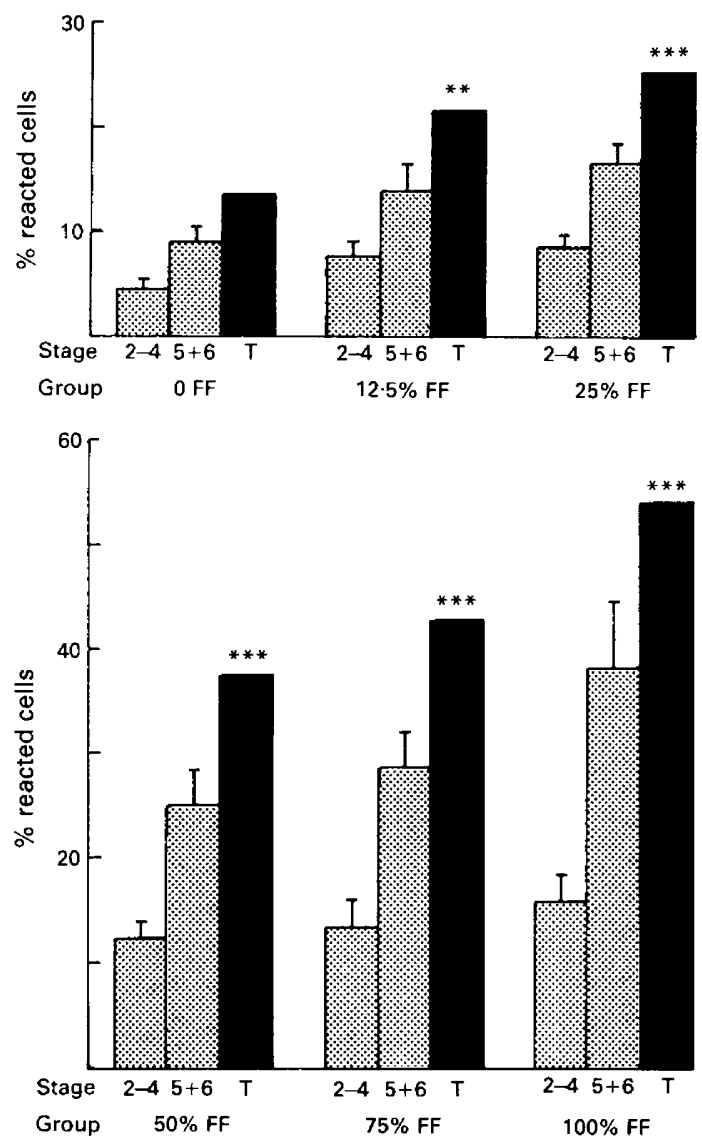

Fig. 4. AR response in human sperm suspensions $(n=7)$ prepared by swim-up and incubated for $24 \mathrm{~h}$ in increasing concentrations of human $\mathrm{FF} .{ }^{* *} P<0.01,{ }^{* * *} P<0.001$ compared with FF-free suspensions.

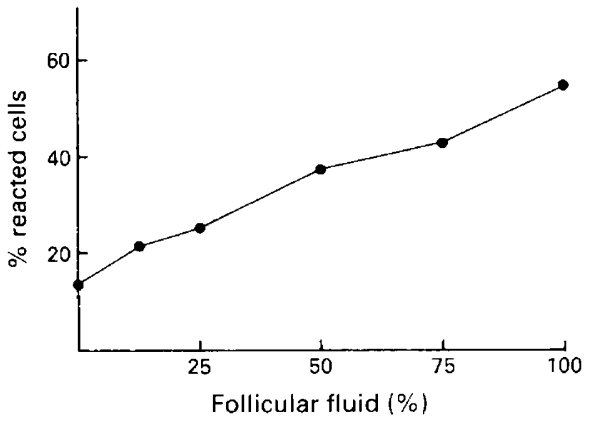

Fig. 5. Mean percentages of cells at Stages $2-6$ of the AR after $24 \mathrm{~h}$ incubation in increasing concentrations of human FF. 


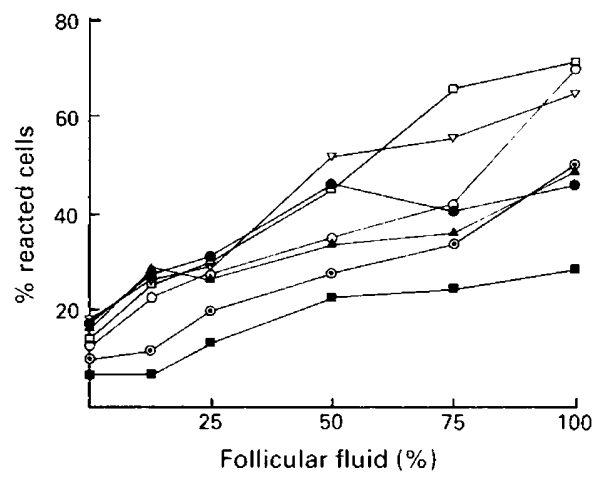

Fig. 6. AR response in 7 individual human sperm suspensions incubated in increasing concentrations of human FF for $24 \mathrm{~h}$. Each point represents the total proportion of cells at Stages 2-6.

those in the other two; this reflected a higher number of cells at both intermediate and fully reacted stages. Motility at $>90 \%$ was maintained in all groups over the 25 -h period.

\section{Series $I I$}

Motile spermatozoa selected by a different method were incubated for a total of $25 \mathrm{~h}$ under the same 3 sets of conditions used in Series I. Although the level of response was higher than that in Series I, the pattern (Fig. 3) was very similar: no significant difference between control $(22 \cdot 0 \%$ reacted cells) and control $+1 \mathrm{~h}$ FF $(23.8 \%)$ groups, but a significantly increased response $(P<0.001)$ in the continuous FF group $(52.5 \%)$. Again, motility at $>90 \%$ was maintained.

\section{Series $I I I$}

Motile spermatozoa were incubated for $24 \mathrm{~h}$ continuously in medium containing $0,12.5 \%$, $25 \%, 50 \%, 75 \%$ or $100 \% \mathrm{FF}$. The proportions of reacted cells after $24 \mathrm{~h}$ were $13 \cdot 7 \%, 21 \cdot 7 \%$, $25 \cdot 3 \%, 37 \cdot 5 \%, 42.9 \%$ and $54 \cdot 3 \%$, respectively (Fig. 4). The presence of FF significantly increased the response at all concentrations $(P<0.01$ for $12.5 \% \mathrm{FF} ; P<0.001$ for $25-100 \% \mathrm{FF})$. In all groups, the majority of responding cells were fully reacted although there was also some increase in the numbers of cells at intermediate stages. When the mean value for reacted cells was plotted against FF concentration, a linear response was obtained (Fig. 5), indicating a consistent concentration-dependent AR response to FF. Values from the individual samples showed this same general pattern, i.e. the mean values were not distorted by just 1 or 2 of the 7 suspensions examined (Fig. 6). Motility was maintained at $>90 \%$ in all samples.

\section{Follicular fluid samples}

In each series several FF samples were assessed and in several instances, the same sample was used with two different sperm preparations. For example, 4 FF samples were used in Series I and another 6 in Series III. Within each Series, responses to all FF samples were similar, demonstrating that properties common to FF in general, rather than a few discrete samples, were responsible.

\section{General ultrastructure}

Ultrastructural examination revealed that the FF-incubated spermatozoa were morphologically normal and similar to those in control suspensions. However, in all 3 series in some of the samples exposed to continuous FF the acrosomes became slightly swollen and electron opaque, 
although the membranes were intact. Since these cells did not exhibit the criteria we have used for classification of the intermediate Stage 2, we have included them with the Stage 1, acrosomeunreacted, spermatozoa. In Series III, with increasing concentrations of FF, some individuals did not have any of these spermatozoa, even in the $100 \%$ FF samples, while others had them at the lowest concentration $(12 \cdot 5 \%)$ used.

\section{Discussion}

The results of the present study indicate that human follicular fluid can significantly stimulate the AR in human spermatozoa, but only when present continuously for an extended period ( $>6 \mathrm{~h}$ ). A relatively low incidence of spontaneous AR $(\sim 15 \%)$ over a $24-25$-h incubation time was obtained in control suspensions incubated continuously in Earle's medium, which supports successful human in-vitro fertilization. This is consistent with our previously reported results using semen samples from men of proven fertility (Stock \& Fraser, 1987). In the present experiments, the introduction of $50 \% \mathrm{FF}$ for $1 \mathrm{~h}$ after 5 and $24 \mathrm{~h}$ incubation in control medium failed to elicit any detectable increase in $\mathrm{AR}$; the number of reacted cells was indistinguishable from that in control suspensions. In contrast, the continuous presence of $50 \%$ FF slightly increased the response by $6 \mathrm{~h}$ and, after $25 \mathrm{~h}$, significantly elevated the number of reacted cells to more than twice that in either the control or control $+1 \mathrm{~h}$ FF groups. This pattern of response, with only continuous FF producing a significant effect, was obtained with both methods of sperm preparation evaluated, namely swim-up directly from semen (Series I) or from a washed pellet (Series II). The level of response was higher in Series II, possibly reflecting the extended contact with seminal plasma. Cheng (1985) has demonstrated that initial incubation of boar spermatozoa in seminal plasma at room temperature resulted in improved in-vitro fertilization results, possibly due to removal of decapacitation factors from spermatozoa by enzymes in seminal plasma. Finally, when spermatozoa were incubated for $24 \mathrm{~h}$ in increasing concentrations of FF, a linear, dose-dependent response was observed. All FFtreated suspensions had significantly increased numbers of reacted cells, compared with FF-free control samples.

Our results, which provide no evidence that FF can trigger a rapid AR response in capacitated human spermatozoa, are in direct contrast to several recently published studies. Tesařík (1985) reported that a 1-h incubation in both human FF and cumulus cells could promote the AR in spermatozoa preincubated for 5 and $15 \mathrm{~h}$. However, in 3 of the 5 samples observed at $15 \mathrm{~h}$ there was a marked decline in motility, which could indicate that many cells were dead and possibly degenerating. Follicular fluid and fractions obtained after gel filtration of FF have been reported to elicit rapid (within 3-20 min) AR after $6 \mathrm{~h}$ and $24 \mathrm{~h}$ preincubation (Suarez et al., 1986; Yudin et al., 1988; Siiteri et al., 1988), although no response to whole FF was observed with preincubation for less than $6 \mathrm{~h}$ (Suarez et al., 1986). In contrast, Mortimer \& Camenzind (1989) reported results similar to ours: preincubation of sperm suspensions for $6 \mathrm{~h}$ followed by a 2-h exposure to concentrations of up to $100 \% \mathrm{FF}$ failed to trigger a marked AR response. The reasons for these discrepancies are not immediately obvious. While basic protocols have varied somewhat, e.g. method of sperm preparation, amount and source of albumin, it is difficult to ascribe the differences in response to any one factor. For example, Suarez et al. (1986) and Mortimer \& Camenzind (1989) both used Percoll gradients to prepare sperm suspensions but obtained different results. Suarez et al. (1986) used a variety of purified albumins, both human and bovine, but there is no evidence that the human AR is supported or promoted to a greater extent by these preparations than by those which we and others have used. If FF acts as the trigger of the AR in vivo, as has been suggested (Suarez et al., 1986), this is a very important, fundamental biological attribute and should be detectable in a system demonstrably able to support in-vitro fertilization. Therefore we would have expected to observe a rapid response to $\mathrm{FF}$ in suspensions preincubated for $24 \mathrm{~h}$, and yet we did not. Mortimer \& Camenzind (1989) have suggested that the methods used by Suarez et al. (1986) to permeabilize 
the air-dried spermatozoa before indirect immunofluorescence assessment for presence/absence of the acrosome may have damaged the acrosome and have resulted in increased false positive assessments. Further evaluation is needed to resolve these conflicting results.

Continuous exposure of spermatozoa to increasing concentrations of FF for $24 \mathrm{~h}$ in the present study produced a dose-dependent linear increase in AR (Fig. 4), the response being significant for all concentrations. An increase in fully reacted cells was the main contributor to this, although there was also a steady rise in the intermediate stages. In contrast, Suarez et al. (1986) observed little difference in the response to a 5-fold range of FF concentrations and Siiteri et al. (1988) observed no differences over a 4-fold range of FF fraction concentrations. However, studies in other species (hamster: Yanagimachi, 1969b; rabbit: Oliphant, 1976; Oliphant et al., 1977) showed AR inducing-activity of FF to be concentration-dependent, as observed here.

In an earlier study (Stock \& Fraser, 1987) we reported that ultrastructural examination of spermatozoa from men of proven fertility incubated in capacitating conditions over a $24-\mathrm{h}$ period revealed an AR that differed from the generalized mammalian response. Although typical initial, acrosome-intact and final, acrosome-reacted spermatozoa are observed, the intermediate stage involving fusion of the plasma and outer acrosomal membranes to form hybrid vesicles is not seen. Instead, intermediate stages exhibit vesicles, formed primarily from the outer acrosomal membranes, within apparently intact plasma and outer acrosomal membranes (Fig. 1b). In the present study the morphological changes observed in human spermatozoa incubated with and without FF were indistinguishable from those previously reported both by us and others (Nagae et al., 1986; Tesarík et al., 1988). We have also seen the same intermediate stages in sperm samples treated with an inophore and fixed within $15 \mathrm{~min}$ while highly motile, thereby excluding the possibility that these stages simply represent degenerative changes in moribund cells (unpublished observations). However, Yudin et al. (1988) have indicated that a human FF fraction promotes changes in human spermatozoa consistent with the classical AR. The post-fixation for electron microscopy employed in their study differed from techniques usually used, but this is unlikely to explain the contradictory observations which need to be further investigated.

In our study, motility was maintained at $>90 \%$, even when cells were incubated continuously in $100 \%$ unheated human FF. However, numerous conflicting reports regarding the effects of FF on sperm motility and the possible necessity of heating FF to destroy factors (e.g. complement) capable of immobilizing spermatozoa exist in the literature. The first report of a deleterious effect of human FF on human spermatozoa was made by Mukerjee \& Lippes (1972), who observed a very marked decline in motility unless FF was heated before use. More recently, Langlais et al. (1988) have reported that a low concentration $(10 \%)$ of unheated FF was toxic to human spermatozoa during a 2-h incubation, although heating abolished this effect. Mortimer \& Camenzind (1989) found that incubation of spermatozoa for $2 \mathrm{~h}$ in increasing concentrations of heated FF, following a 6-h preincubation, resulted in a negative correlation between motility and FF (up to $100 \%$ ). Suarez et al. (1986) observed a small decline in motility after addition of unheated human FF, but since heating did not consistently reverse this effect, the responses did not appear to be due to the action of complement. Tesařík (1985) found no deleterious effect with fresh, unheated FF. Similarly, Yell \& Cummings (1988) showed that a brief exposure of preincubated spermatozoa to unheated human FF had no observable negative effect and, in fact, increased the penetration rate into zona-free hamster oocytes. This wide range of responses to FF is bewildering and difficult to resolve readily; presumably methods used for recovery/treatment/storage of FF before use, as well as sperm suspension preparation, are important. All our FF samples were obtained from women with at least some oocytes which were successfully fertilized in vitro. The fact that we found no deleterious effect of unheated FF on motility, a commonly used parameter of sperm 'well-being', leads us to believe that our observations are valid. Indeed, the reported inhibitory effects of FF are somewhat puzzling, considering that spermatozoa are exposed to unheated FF at the site of fertilization in vivo. Although the fluid may be dispersed, the volume released at ovulation could result in spermatozoa being bathed in a relatively high concentration of FF. 
While we have made no attempt to identify the molecular species capable of exerting a positive effect on occurrence of the AR, there have been numerous studies designed to do so. Yanagimachi (1969a) reported that bovine $\mathrm{FF}$, after heating to $56^{\circ} \mathrm{C}$, contained two factors: the first, which was dialysable and heat stable, being responsible for stimulating motility and the second, which was non-dialysable and heat labile (at $90^{\circ} \mathrm{C}$ ) being responsible for AR induction. Similarly, Oliphant $e t$ al. (1977) noted that heating human FF resulted in a significantly reduced incidence of AR in rabbit spermatozoa. There is some evidence that albumin, an abundant component of FF, has positive effects on the AR. Lui et al. (1977) identified the AR-inducing component in bovine FF as albumin and we have shown that albumin, while not obligatory for capacitation, is required for the AR to occur in mouse spermatozoa (Fraser, 1985). Langlais \& Roberts (1985) and Langlais et al. (1988) have suggested that albumin in human FF promotes capacitation of human spermatozoa by acting as a sterol acceptor and thus stimulating cholesterol efflux from sperm membranes. A decrease in the cholesterol:phospholipid molar ratio of the sperm plasma membrane during capacitation, leading to increased membrane permeability to extracellular calcium, has been postulated by Davis (1978). Langlais et al. (1988) found that FF albumin has a sterol binding capacity 2-3 times greater than serum albumin. However, Suarez et al. (1986) and Siiteri et al. (1988) have reported that the active fraction isolated from human FF eluted after the albumin and hence represented a different component. Furthermore, neither incubation of sperm suspensions for $10 \mathrm{~h}$ in medium containing $26 \mathrm{mg}$ albumin $/ \mathrm{ml}$ nor addition of serum from $\mathrm{FF}$ donors after $10 \mathrm{~h}$ sperm preincubation stimulated the AR response. In a preliminary study, we have also failed to detect an elevated AR response when suspensions were incubated for $24 \mathrm{~h}$ in $26 \mathrm{mg} \mathrm{hSA} / \mathrm{ml}$ compared with the usual $5 \mathrm{mg} /$ $\mathrm{ml}$ (unpublished observations).

Glycosaminoglycans (GAGs) are other possible candidates for the active factor. FF is a rich source of chondroitin sulphate and heparin sulphate, and cumulus cells manufacture hyaluronic acid during expansion of the cumulus (Ball et al., 1987). Numerous studies have shown that GAGs can stimulate the AR, at least in some species (e.g. Meizel, 1985; Meizel \& Turner, 1986), although Siiteri et al.(1988) were unable to find supporting evidence for this with human spermatozoa. Heparin, which promotes fertilization in vitro of bovine oocytes, has been shown to affect capacitation of bovine spermatozoa, but not to trigger the AR (Parrish et al., 1988). Boyers et al. (1987) found that heparin added to FF at the time of human oocyte recovery had no effect on fertilization or the AR. It is clear that further evaluation of GAGs is required before these various responses can be resolved, if indeed that is possible. At present the active components of FF remain uncharacterized, although Siiteri et al. (1988) have some evidence that the active fraction in human FF has an approximate weight of 50000 , with some AR-inducing activity being dialysable and some non-dialysable.

In conclusion we have demonstrated that human FF can increase the proportion of spermatozoa undergoing the AR in a concentration-dependent manner, but only after a long continuous exposure. We find no evidence that FF can trigger a rapid response in spermatozoa, despite prolonged preincubation in conditions which support capacitation and fertilization in vitro. This suggests that FF on its own is unlikely to play a controlling role in fertilization; however, it might act synergistically with initiator(s) to maximize the chances of successful fertilization.

We thank Paul Fielding for expert technical assistance; Andrew Osborne for artwork; Leon Kelberman for photography; and Sarah Fuge and Mary Cheesewright for secretarial assistance. This study was supported by a grant from Birthright to L.R.F.

\section{References}

Austin, C.R. (1951) Observations on the penetration of the sperm into the mammalian egg. Aust. J. Sci. Res. B4, 581-589.

Ball, G.D., Bellin, M.E., Ax, R.L. \& First, N.L. (1982) Glycosaminoglycans in bovine cumulus-oocyte complexes: morphology and chemistry, Molec. cell. Endocrinol. 28, 113-122.

Bedford, J.M. (1970) Sperm capacitation and fertilization in mammals. Biol. Reprod., Suppl. 2, 128-158. 
Bedford, J.M. (1983) Significance of the need for sperm capacitation before fertilization in eutherian mammals. Biol. Reprod. 28, 108-120.

Boyers, S.P., Tarlazis, B.C., Stronk, J.N. \& Decherney, A.H. (1987) Fertilization and cleavage rates of heparin-exposed human oocytes in vitro, and the effect of heparin on the acrosome reaction. Fert. Steril. 48, 628-632.

Chang, M.C. (1951) Fertilizing capacity of spermatozoa deposited into the Fallopian tube. Nature, Lond. 168, 697-698.

Cheng, W.T.-K. (1985) In vitro Fertilization of Farm Animal Oocytes. Ph.D. thesis, CNAA, Cambridge.

Davis, B.K. (1978) Inhibition of fertilizing capacity in mammalian spermatozoa by natural and synthetic vesicles. In Symposium of the Pharmalogical Effects of Lipids, pp. 145-157. Am. Oil Chem. Soc. Monograph No. 5 (Champaign, IL).

Edwards, R.G. (1974) Follicular fluid. J. Reprod. Fert. 37, I89-220.

Fraser, L.R. (1985) Albumin is required to support the acrosome reaction but not capacitation in mouse spermatozoa in vitro. J. Reprod. Fert. 74, 185-196.

Fraser, L.R. \& Ahuja, K.K. (1988) Metabolic and surface events in fertilization. Gamete Res. 20, 491-519.

Langlais, J. \& Roberts, K.D. (1985) A molecular membrane model of sperm capacitation and the acrosome reaction of mammalian spermatozoa. Gamete Res. 12, 183-224.

Langlais, J., Kan, F.W.K., Granger, L., Raymond, L., Blease, G. \& Roberts, K.D. (1988) Identification of sterol acceptors that stimulate cholesterol efflux from human spermatozoa during in vitro capacitation. Gamete Res. 20, 185-201.

Lui, C.W., Cornett, L.E. \& Meizel, S. (1977) Identification of the bovine follicular fluid protein involved in the in vitro induction of the hamster sperm acrosome reaction. Biol. Reprod. 17, 3441.

Meizel, S. (1985) Molecules that initiate or help stimulate the acrosome reaction by their interaction with the mammalian sperm surface. Am. J. Anat. 174, 285302.

Meizel, S. \& Turner, K.O. (1986) Glycosaminoglycans stimulate the acrosome reaction of previously capacitated hamster sperm. J. exp. Zool. 237, 137-139.

Mortimer, D. \& Camenzind, A.R. (1989) The role of follicular fluid in inducing the acrosome reaction in human spermatozoa incubated in vitro. Human Reprod. 4, 169-174.

Mukerjee, A.B. \& Lippes, J. (1972) Effect of human follicular fluid and tubal fluids on human, mouse and rat spermatozoa in vitro. Can. J. Genet. Cytol. 14, 167174.

Nagae, T., Yanagimachi, R., Srivastava, P.N. \&
Yanagimachi, H. (1986) Acrosome reaction in human spermatozoa. Fert. Steril. 45, 701-707.

Oliphant, G. (1976) Removal of sperm-bound seminal plasma components as a prerequisite to induction of the rabbit acrosome reaction. Fert. Steril. 27, 28-38.

Oliphant, G., Cabot, C.L. \& Singhas, C.A. (1977) Nature of the rabbit acrosome reaction-inducing activity of follicular fluid. J. Reprod. Fert. 50, 245-250.

Parrish, J.J., Susko-Parrish, J., Winer, M.A. \& First, N.L. (1988) Capacitation of bovine sperm by heparin. Biol. Reprod. 38, 1171-1180.

Siiteri, J.E., Gottlieb, W. \& Meizel, S. (1988) Partial characterization of a fraction from human follicular fluid that initiates the human sperm acrosome in vitro. Gamete Res. 20, 25-42.

Snedecor, G. \& Cochran, W. (1967) Statistical Methods, 6 th edn. Iowa State University Press, Ames.

Stock, C.E. \& Fraser, L.R. (1987) The acrosome reaction in human sperm from men of proven fertility. Human Reprod. 2, 109-119.

Stock, C.E., Bates, R., Lindsay, K.S., Edmonds, D.K. \& Fraser, L.R. (1988) Enhancement of the human acrosome reaction by follicular fluid. J. Reprod. Fert., Abstr. Ser. 1, 39, Abstr.

Suarez, S.S., Wolf, D.P. \& Meizel, S. (1986) Induction of the acrosome reaction in human spermatozoa by a fraction of human follicular fluid. Gamete Res. 14, $107-121$.

Tesařík, J. (1985) Comparison of acrosome reactioninducing activities of human cumulus oophorus, follicular fluid and inophore A23187 in human sperm populations of proven fertilizing ability in vitro. $J$. Reprod. Fert. 74, 383-388.

Tesařík, J., Drahorád, J. \& Pěknicová, J. (1988) Subcellular immunochemical localization of acrosin in human spermatozoa during the acrosome reaction and zona pellucida penetration. Fert. Steril. 50, 133-141.

Yanagimachi, R. (1969a) In vitro acrosome reaction and capacitation of golden hamster spermatozoa by bovine follicular fluid and its fractions. J. exp. Zool. 170, $269-280$.

Yanagimachi, R. (1969b) In vitro capacitation of hamster spermatozoa by follicular fluid. J. Reprod. Fert. 18, 275-286.

Yell, B. \& Cummings, M.S. (1988) Modification of the sperm penetration assay using human follicular fluid to minimize false negative results. Fert. Steril 50, $123-128$.

Yudin, A.I., Gottlieb, W. \& Meizel, S. (1988) Ultrastructural studies of the early events of the human sperm acrosome reaction as initiated by human follicular fluid. Gamete Res. 20, 11-24.

Received 8 November 1988 